\title{
ON SOME HOLOMORPHIC DYNAMICAL SYSTEMS
}

\author{
WOJCIECH CHOJNACKI
}

\section{Introduction}

Let $D$ be a domain in $\mathbb{C}^{N}$ and $\varphi$ a holomorphic automorphism of $D$. Let $\mathscr{C}$ be the measure class of the Lebesgue measure in $D$, i.e., the set of all positive regular Borel measures on $D$ whose null sets coincide with the Lebesgue null sets. Let $\varphi_{*}$ be the automorphism of $\mathscr{C}$ given by

$$
\left(\varphi_{*} \mu\right)(B)=\mu\left(\varphi^{-1}(B)\right) \quad(\mu \in \mathscr{C}, B \in \mathscr{B}(D)),
$$

where $\mathscr{B}(D)$ denotes the Borel $\sigma$-algebra of $D$. Adopting the terminology introduced in [4], we will say that $\varphi_{*}$ is finite if it has a fixed point among probability measures.

Let $L^{2} H(D)$ be the Hilbert space of all square Lebesgue integrable holomorphic functions on $D$. Suppose that $L^{2} H(D) \neq\{0\}$. Let $U_{\varphi}$ be the unitary operator in $L^{2} H(D)$ defined by

$$
U_{\varphi} f=(f \circ \varphi) J_{\varphi} \quad\left(f \in L^{2} H(D)\right)
$$

where

$$
J_{\varphi}=\operatorname{det}\left(\frac{\partial \varphi_{i}}{\partial z_{j}}\right)_{1 \leq i, j \leq N} \quad\left(\varphi=\left(\varphi_{1}, \ldots, \varphi_{N}\right)\right) .
$$

The purpose of this paper is to exhibit various relations between $\varphi, \varphi_{*}$, and $U_{\varphi}$. A fundamental result is that $U_{\varphi}$ has either pure point spectrum or purely continuous spectrum, the first case occurring exactly when $\varphi_{*}$ is finite. We prove that any of the following two conditions ensures the finiteness of $\varphi_{*}$ : $1^{\text {o }}$ the existence of a $\varphi$-invariant probability measure absolutely continuous with respect to Lebesgue measure; $2^{\circ}$ the existence of a relatively compact orbit of $\varphi$. Of course, the first condition is also necessary. We show the necessity of a stronger version of the second condition (embeddability of $\varphi$ in a compact transformation group) provided some mild restrictions on $D$ are imposed. Assuming some hypotheses on $D$, we prove also that if a point in $D$ is wandering, then $U_{\varphi}$ has purely absolutely continuous spectrum, and, conversely, if $U_{\varphi}$ has a non-zero absolutely continuous component in the spectrum, then all points in $D$ are wandering. In particular, the spectrum of $U_{\varphi}$ is either pure point, or purely absolutely continuous, or purely singular continuous. We show that if $D$ is in a class of domains containing among others all bounded analytic polyhedra, then the spectrum of $U_{\varphi}$ cannot be purely singular continuous.

\section{A purity theorem}

The starting point of our discussion is the following

Theorem 1.1. Suppose there exists a $\varphi$-invariant probability measure $\mathbb{P}$ that is absolutely continuous with respect to Lebesgue measure. Then $U_{\varphi}$ has pure point spectrum.

Proof. Given $k \in \mathbb{Z}$ and a bounded Borel measure $\mu$ on the unit circle $\mathbb{T}$, let $\hat{\mu}(k)$ be the $k$ th Fourier coefficient of $\mu$, i.e.,

$$
\hat{\mu}(k)=\int_{\mathbb{T}} t^{-k} \mathrm{~d} \mu(t) .
$$


By the spectral theorem for unitary operators, there is a unique projection-valued measure $P$ on $\mathbb{T}$, taking values in a Boolean algebra of projections in $L^{2} H(D)$, such that for each $k \in \mathbb{Z}$,

$$
U_{\varphi}^{k}=\int_{\mathbb{T}} t^{-k} \mathrm{~d} P(t)
$$

where the integral is to be interpreted in the sense of strong convergence. For any $f, g \in L^{2} H(D)$, let $\pi_{f, g}$ be the complex measure on $\mathbb{T}$ such that

$$
\pi_{f, g}(B)=(P(B) f, g) \quad(B \in \mathscr{B}(\mathbb{T})),
$$

where $(\cdot, \cdot)$ stands for the scalar product in $L^{2} H(D)$. Given $k \in \mathbb{N}$, let

$$
\varphi^{k}=\underbrace{\varphi \circ \cdots \circ \varphi}_{k \text { times }}, \quad \varphi^{-k}=\underbrace{\varphi^{-1} \circ \cdots \circ \varphi^{-1}}_{k \text { times }},
$$

and let $\varphi^{0}$ stand for the identity map of $D$. Since $U_{\varphi}^{k}=U_{\varphi^{k}}$ for each $k \in \mathbb{Z}$, it follows from (1.1) that

$$
\left(U_{\varphi}^{k} f, g\right)=\hat{\pi}_{f, g}(-k)
$$

Let

$$
L^{2} H(D)=H_{p p} \oplus H_{c}
$$

be the orthogonal decomposition of $L^{2} H(D)$ in which $H_{p p}$ is the closure of the linear span of the eigenvectors of $U_{\varphi}$, and $H_{c}$ consists of those $f \in L^{2} H(D)$ for which $\hat{\pi}_{f, f}$ is a continuous measure. As is known, this decomposition reduces $P$.

Suppose that the spectrum of $U_{\varphi}$ is not pure point, that is, there exists a nonzero $f$ in $H_{c}$. Let $g$ be any element of $L^{2} H(D)$ with the decomposition $g=g^{\prime}+g^{\prime \prime}$ with respect to $H_{p p}$ and $H_{c}$. Clearly, $\pi_{f, g}=\pi_{f, g^{\prime \prime}}$. Since

$$
\pi_{f, g^{\prime \prime}}=\frac{1}{4} \sum_{k=0}^{3} i^{k} \pi_{f+i^{k} g^{\prime \prime}, f+i^{k} g^{\prime \prime}}
$$

the measure $\pi_{f, g}$ is continuous. By a theorem of Wiener [17, p. 108],

$$
\lim _{n \rightarrow \infty} \frac{1}{2 n+1} \sum_{k=-n}\left|\hat{\pi}_{f, g}(k)\right|^{2}=\sum_{t \in \mathbb{T}}\left|\pi_{f, g}(\{t\})\right|^{2}=0 .
$$

Thus in view of $(1.2)$

$$
\lim _{n \rightarrow \infty} \frac{1}{2 n+1} \sum_{k=-n}^{n}\left|\left(U_{\varphi}^{k} f, g\right)\right|^{2}=0 .
$$

Let $\left\{\varphi_{n}: n \in \mathbb{N}\right\}$ be any complete orthonormal set in $L^{2} H(D)$. The function

$$
K_{D}(z, w)=\sum_{n=1}^{\infty} \varphi_{n}(z) \overline{\varphi_{n}(w)} \quad(z, w \in D),
$$

called the Bergman function of $D$, does not depend on the particular choice of $\left\{\varphi_{n}: n \in \mathbb{N}\right\}$ (cf. $\left[\mathbf{1}\right.$, p. 21]). For each $z \in D$, let $\chi_{z}$ be the element of $L^{2} H(D)$ such that

$$
\chi_{z}=K_{D}(w, z) \quad(w \in D) .
$$

A fundamental property of $K_{D}$ is that, given $f \in L^{2} H(D)$ and $z \in D$,

$$
f(z)=\left(f, \chi_{z}\right) \text {. }
$$

Let $K$ be a compact subset of $D$ such that $\mathbb{P}(K)>0$. It is well known that

$$
\sup \left\{\left\|\chi_{z}\right\|: z \in K\right\} \leq \pi^{-N / 2} \operatorname{dist}(K, \partial D)^{-1},
$$


where $\|\cdot\|$ stands for the norm in $L^{2} H(D)$ and the distance $\operatorname{dist}(K, \partial D)$ between $K$ and the boundary $\partial D$ of $D$ refers to the supremum norm in $\mathbb{C}^{N}$. From this estimate it follows that the restrictions to $K$ of the functions

$$
z \mapsto \frac{1}{2 n+1} \sum_{k=-n}^{n}\left|\left(U_{\varphi}^{k} f, g\right)\right|^{2} \quad(n \in \mathbb{N})
$$

are uniformly bounded. On account of (1.4), we find that

$$
\lim _{n \rightarrow \infty} \frac{1}{2 n+1} \sum_{k=-n}^{n} \int_{K}\left|\left(U_{\varphi}^{k} f, g\right)\right|^{2} \mathrm{~d} \lambda(z)=0,
$$

where $\lambda$ stands for the Lebesgue measure in $D$. By (1.7) for each $k \in \mathbb{Z}$,

$$
\int_{K}\left|\left(U_{\varphi}^{k} f, g\right)\right|^{2} \mathrm{~d} \lambda(z)=\int_{K}\left|\left(f \circ \varphi^{k}\right) J_{\varphi^{k}}\right|^{2} \mathrm{~d} \lambda=\int_{\varphi^{k}(K)}|f|^{2} \mathrm{~d} \lambda,
$$

so if we denote by $1_{B}$ the characteristic function of a subset $B$ of $D$, then (1.9) can be rewritten in the form

$$
\lim _{n \rightarrow \infty}\left(\frac{1}{2 n+1} \sum_{k=-n}^{n} 1_{\varphi^{k}(K)}\right)|f|^{2} \mathrm{~d} \lambda=0 .
$$

As $f \neq 0 \lambda$-a.e., the last identity and Fatou's lemma imply

$$
\liminf _{n \rightarrow 0} \frac{1}{2 n+1} \sum_{k=-n}^{n} 1_{\varphi^{k}(K)}=0 \quad \lambda \text {-a.e. }
$$

Let $\mathfrak{M}$ be the $\sigma$-algebra $\left\{B \in \mathscr{B}(D): 1_{B}=1_{\varphi(B)} \mathbb{P}\right.$-a.e. $\}$ and $\mathbb{E}^{\mathfrak{M}}$ be the corresponding conditional expectation operator. By Birkhoff's ergodic theorem $[\mathbf{1 4}$, p. 25]

$$
\lim _{n \rightarrow 0} \frac{1}{2 n+1} \sum_{k=-n}^{n} 1_{\varphi^{k}(K)}=\mathbb{E}^{\mathfrak{M}}\left(1_{K}\right) \quad \mathbb{P} \text {-a.e. }
$$

Comparing this equality with (1.11) and taking into account that $\mathbb{P}$ is absolutely continuous with respect to $\lambda$, we find that

$$
\mathbb{E}^{\mathfrak{M}}\left(1_{K}\right)=0 \quad \mathbb{P} \text {-a.e. }
$$

Hence

$$
\mathbb{P}(K)=\mathbb{E}\left[\mathbb{E}^{\mathfrak{M}}\left(1_{K}\right)\right]=0,
$$

a contradiction.

The proof is complete.

Theorem 1.2. If $U_{\varphi}$ has a non-zero eigenvector, then $\varphi_{*}$ is finite.

Proof. Let $h$ be an eigenvector of $U_{\varphi}$ of unit norm. Setting

$$
\mathbb{P}(B)=\int_{B}|h|^{2} \mathrm{~d} \lambda \quad(B \in \mathscr{B}(D))
$$

defines a $\varphi$-invariant probability measure on $D$. Since $h \neq 0 \lambda$-a.e., it follows that $\mathbb{P} \in \mathscr{C}$.

The proof is complete.

As a corollary to Theorems 1.1 and 1.2, we obtain the following generalization of a result of $[\mathbf{1 1}]$.

Theorem 1.3. The spectrum of $U_{\varphi}$ is either pure point or purely continuous according as $\varphi_{*}$ is finite or not.

We close this section with two simple examples. 
Example 1.4. Let $D$ be an open ball in $\mathbb{C}^{N}$ centered at 0 and let $\varphi$ be defined as

$$
\varphi\left(z_{1}, \ldots, z_{N}\right)=\left(\mathrm{e}^{i \theta_{1}} z_{1}, \ldots, \mathrm{e}^{i \theta_{N}} z_{N}\right) \quad\left(\left(z_{1}, \ldots, z_{N}\right) \in D\right),
$$

where $\theta_{1}, \ldots, \theta_{N} \in \mathbb{R}$. Then the normalized Lebesgue measure in $D$ is $\varphi$-invariant, and so, by Theorem $1.3, U_{\varphi}$ has pure point spectrum.

Example 1.5. Let $D=\left\{\left(z_{1}, \ldots, z_{N}\right) \in \mathbb{C}^{N}: \operatorname{Im} z_{1}>0, \ldots, \operatorname{Im} z_{N}>0\right\}$ and let $\varphi$ be defined as

$$
\varphi\left(z_{1}, \ldots, z_{N}\right)=\left(z_{1}+t_{1}, \ldots, z_{N}+t_{N}\right) \quad\left(\left(z_{1}, \ldots, z_{N}\right) \in D\right)
$$

where $\left(t_{1}, \ldots, t_{N}\right) \in \mathbb{R}^{N} \backslash\{(0, \ldots, 0)\}$. Let $U$ be an open ball in $D$ with sufficiently small radius so that $\varphi^{m}(U) \cap \varphi^{n}(U)=\emptyset$ for any distinct integers $m$ and $n$. Suppose that there exists a $\varphi$-invariant probability measure $\mathbb{P} \in \mathscr{C}$. If $\mathscr{N}$ is any finite subset of $\mathbb{Z}$ with cardinality $n$, then

$$
n \cdot \mathbb{P}(U)=\sum_{m \in \mathscr{N}} \mathbb{P}\left(\varphi^{m}(U)\right)=\mathbb{P}\left(\bigcup_{m \in \mathscr{N}} \varphi^{m}(U)\right) \leq 1 .
$$

Hence $\mathbb{P}(U)=0$, which is incompatible with $\mathbb{P} \in \mathscr{C}$. Therefore $\varphi_{*}$ is not finite, and consequently, by Theorem $1.3, U_{\varphi}$ has purely continuous spectrum.

\section{Pure point spectrum}

For each $w \in D$, let $\mathscr{O}(w)$ denote the orbit $\left\{\varphi^{k}(w): k \in \mathbb{Z}\right\}$ of $w$.

The following theorem generalizes some results of $[\mathbf{1 1}, \mathbf{1 2}, \mathbf{1 3}]$.

Theorem 2.1. Suppose that there exists $w \in D$ such that the closure of $\mathscr{O}(w)$ in $D$ is compact. Then $U_{\varphi}$ has pure point spectrum.

Proof. Given a multiindex $\alpha=\left(\alpha_{1}, \ldots, \alpha_{N}\right) \in(\mathbb{N} \cup\{0\})^{N}$, let $|\alpha|=\alpha_{1}+\cdots+\alpha_{N}$, and, given $t \in D$, let $\bar{\partial}^{\alpha} \chi_{t}$ or $\partial_{\bar{z}_{1}^{\alpha_{1}} \ldots \bar{z}_{N}^{\alpha_{N}}}$ denote the function

$$
\left.w \mapsto \frac{\partial^{|\alpha|} K_{D}}{\partial \bar{z}_{1}^{\alpha_{1}} \cdots \partial \bar{z}_{N}^{\alpha_{N}}}(w, z)\right|_{z=t} .
$$

It is easily seen that for each $\alpha \in(\mathbb{N} \cup\{0\})^{N}$ and each $z \in D, \bar{\partial}^{\alpha} \chi_{z}$ is in $L^{2} H(D)$ and

$$
\frac{\partial^{|\alpha|} f}{\partial z_{1}^{\alpha_{1}} \cdots \partial z_{N}^{\alpha_{N}}}(z)=\left(f, \bar{\partial}^{\alpha} \chi_{z}\right) \quad\left(f \in L^{2} H(D)\right) .
$$

Since $L^{2} H(D) \neq\{0\}$, it follows immediately from the last equality that for each $z \in D$ there is a multiindex $\alpha$ such that $\bar{\partial}^{\alpha} \chi_{z} \neq 0$.

Let $\alpha$ be a multiindex such that $\bar{\partial}^{\alpha} \chi_{w} \neq 0$ and $\bar{\partial}^{\beta} \chi_{w}=0$ for every multiindex $\beta$ with $|\beta|<|\alpha|$. Then

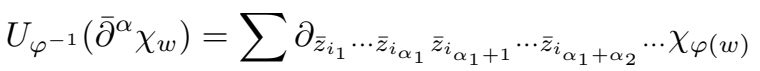

$$
\begin{aligned}
& \times \overline{\frac{\partial \varphi_{i_{1}}}{\partial z_{1}}(w)} \cdots \overline{\frac{\partial \varphi_{i_{\alpha_{1}}}}{\partial z_{1}}(w)} \overline{\frac{\partial \varphi_{i_{\alpha_{1}+1}}}{\partial z_{1}}(w)} \cdots \overline{\frac{\partial \varphi_{i_{\alpha_{1}+\alpha_{2}}}}{\partial z_{1}}(w)} \cdots \\
& \cdot \overline{J_{\varphi}(w)}
\end{aligned}
$$

where the sum extends over all multiindices $\left(i_{1}, \ldots, i_{|\alpha|}\right)$ with $1 \leq i_{1} \leq N, \ldots, 1 \leq$ $i_{|\alpha|} \leq N$. A similar formula is valid with $\varphi$ replaced by $\varphi^{k}(k \in \mathbb{Z} \backslash\{0\}), \alpha$ replaced by $\gamma$ such that $|\gamma|=|\alpha|$, and $w$ replaced by $z \in \mathscr{O}(w)$. In particular, it follows from that formula that the dimension $d(z)$ of the linear span $H_{z}$ of $\left\{\bar{\partial}^{\gamma} \chi_{z}:|\gamma|=|\alpha|\right\}$ takes on a constant value $d$ for $z \in \mathscr{O}(w)$. Obviously, $d(z) \leq d$ for all $z$ in the closure $\overline{\mathscr{O}(w)}$ of $\mathscr{O}(w)$ in $D$. Being compact, $\overline{\mathscr{O}(w)}$ contains a minimal $\varphi$-invariant subset $K$ (cf. [7, Thm. 2.22]). Clearly, if $w^{\prime} \in K$, then $\overline{\mathscr{O}\left(w^{\prime}\right)}=\overline{\mathscr{O}(z)}$ for each $z \in \overline{\mathscr{O}\left(w^{\prime}\right)}$. 
Replacing $w$ by any $w^{\prime}$ in $K$ if necessary, we may safely assume that $\overline{\mathscr{O}(z)}=\overline{\mathscr{O}(w)}$ for each $z \in \mathscr{O}(w)$. Then $d(z)=d$ for all $z \in \overline{\mathscr{O}(w)}$. Now it is clear that $z \mapsto H_{z}$ is a continuous mapping from $\overline{\mathscr{O}(w)}$ into the Grassmannian of $d$-dimensional linear subspaces of $L^{2} H(D)$, and that the image of $\overline{\mathscr{O}(w)}$ by that mapping is compact. Since, for each $k \in \mathbb{Z}, U_{\varphi^{k}}\left(\bar{\partial}^{\alpha} \chi_{w}\right)$ is an element of $H_{\varphi^{-k}(w)}$ of norm $\left\|\bar{\partial}^{\alpha} \chi_{w}\right\|$, it follows that the closure of $\left\{U_{\varphi^{k}}\left(\bar{\partial}^{\alpha} \chi_{w}\right): k \in \mathbb{Z}\right\}$ is compact. Let $H$ be the closure of the linear span of $\left\{U_{\varphi^{k}}\left(\bar{\partial}^{\alpha} \chi_{w}\right): k \in \mathbb{Z}\right\}$. H is a reducing subspace for $U_{\varphi}$ and, by Weyl's theorem [10, p. 456], the unitary representation $k \mapsto U_{\varphi^{k}} \mid H$ of $Z$ in $H$ is a direct sum of finite dimensional unitary representations (here $U_{\varphi^{k}} \mid H$ stands for the restriction of $U_{\varphi^{k}}$ to $H$ ). Since a unitary operator in a finite dimensional complex Hilbert space has pure point spectrum, it follows that $U_{\varphi}$ has an eigenvector. By Theorem $1.3, U_{\varphi}$ actually has pure point spectrum.

The proof is complete.

\section{Property (A)}

We shall say that $(D, \varphi)$ has property (A) if for each $w \in D$, there exist relatively compact open neighbourhoods $U$ and $V$ of $w$ in $D$ such that if $U \cap \varphi^{n}(U) \neq \emptyset$ for some $n \in \mathbb{Z}$, then $\varphi^{n}(U) \subset V$.

Theorem 3.1. Suppose that $D$ is a domain in $\mathbb{C}$ and that $U_{\varphi}$ has pure point spectrum. Then $(D, \varphi)$ has property $(\mathrm{A})$.

Proof. Since $L^{2} H(D) \neq\{0\}$, it follows from a result of Wiegerinck [16] that $L^{2} H(D)$ is in fact infinite dimensional. In particular, there exist two linearly independent eigenvectors $h_{1}, h_{2}$ of $U_{\varphi}$. Let $w$ be a point in $D$. Clearly, at least one of the meromorphic functions $h_{1} / h_{2}$ and $h_{2} / h_{1}$, say $h_{1} / h_{2}$ is holomorphic at $w$. Let $D^{\prime}$ denote $D$ with the poles of $h_{1} / h_{2}$ deleted. For any $\delta>0$, let

$$
W_{\delta}=\left\{z \in D^{\prime}:\left|h_{1}(z) / h_{2}(z)-h_{1}(w) / h_{2}(w)\right|<\delta\right\},
$$

and let $V_{\delta}$ be the (open) component of $W_{\delta}$ containing $w$. As is well known [6, p. 10], there exist $k \in \mathbb{N}$, an open neighbourhood $\Omega^{\prime} \subset D^{\prime}$ of $w$, an open neighbourhood $\Delta$ of 0 , and one-to-one holomorphic functions $g_{1}: \Omega^{\prime} \rightarrow \Delta$ and $g_{2}: \Delta \rightarrow \Delta$ such that

$$
h_{1}(z) / h_{2}(z)-h_{1}(w) / h_{2}(w)=g_{2}\left(\left[g_{1}(z)\right]^{k}\right)
$$

for each $z \in \Omega^{\prime}$. It follows from this representation that there exists $\delta>0$ such that the closure of $V_{3 \delta}$ in $D$ is compact. The proof will be complete once we show that if $V_{\delta} \cap \varphi^{n}\left(V_{\delta}\right) \neq \emptyset$ for some $n \in \mathbb{Z}$, then $\varphi^{n}\left(V_{\delta}\right) \subset V_{3 \delta}$.

Suppose that $\varphi^{n}\left(z_{1}\right)=z_{2}$ for $z_{1}, z_{2} \in V_{\delta}$ and $n \in \mathbb{Z}$. Then, for each $z \in V_{\delta}$,

$$
\begin{aligned}
&\left|h_{1}\left(\varphi^{n}(z)\right) / h_{2}\left(\varphi^{n}(z)\right)-h_{1}(w) / h_{2}(w)\right| \\
& \leq\left|h_{1}\left(\varphi^{n}(z)\right) / h_{2}\left(\varphi^{n}(z)\right)-h_{1}\left(\varphi^{n}\left(z_{1}\right)\right) / h_{2}\left(\varphi^{n}\left(z_{1}\right)\right)\right| \\
&+\left|h_{1}\left(z_{2}\right) / h_{2}\left(z_{2}\right)-h_{1}(w) / h_{2}(w)\right| \\
&=\left|h_{1}(z) / h_{2}(z)-h_{1}\left(z_{1}\right) / h_{2}\left(z_{1}\right)\right|+\left|h_{1}\left(z_{2}\right) / h_{2}\left(z_{2}\right)-h_{1}(w) / h_{2}(w)\right|<3 \delta .
\end{aligned}
$$

Thus $\varphi^{n}\left(V_{\delta}\right) \subset W_{3 \delta}$. Since $\varphi^{n}\left(V_{\delta}\right)$ is connected and intersects $V_{\delta} \subset V_{3 \delta}$, it follows that $\varphi^{n}\left(V_{\delta}\right) \subset V_{3 \delta}$.

The proof is complete.

We shall say that a domain $\Omega$ in $\mathbb{C}^{N}$ has property $\mathscr{A}$ if, for each $w \in \Omega, \chi_{w} \neq 0$ and the set comprising $\chi_{w}$ and all the $\partial_{\bar{z}_{i}} \chi_{w}(1 \leq i \leq N)$ is linearly independent.

Theorem 3.2. Suppose that $D$ has property $\mathscr{A}$ and that $U_{\varphi}$ has pure point spectrum. Then $(D, \varphi)$ has property $(\mathrm{A})$. 
Proof. Let $w$ be a point in $D$. As $\chi_{w} \neq 0$, formulae (1.5) and (1.6) applied to a complete orthonormal set in $L^{2} H(D)$ consisting of eigenvectors of $U_{\varphi}$ show that $h(w) \neq 0$ for some eigenvector $h$ of $U_{\varphi}$. Let $D^{\prime}$ denote $D$ with the zeros of $h$ deleted. For any $\delta>0$, let

$$
W_{\delta}=\left\{z \in D^{\prime}:\left\|(\overline{h(z)})^{-1} \chi_{z}-(\overline{h(w)})^{-1} \chi_{w}\right\|<\delta\right\},
$$

and let $V_{\delta}$ be the component of $W_{\delta}$ containing $w$. Since the set

$$
\left\{\chi_{w}\right\} \cup\left\{\partial_{\bar{z}_{i}} \chi_{w}: 1 \leq i \leq N\right\}
$$

is linearly independent, it follows that an open neighbourhood of $w$ can be diffeomorphically embedded in $L^{2} H(D)$ by the map $z \mapsto(\overline{h(z)})^{-1} \chi_{z}$. Accordingly, we can find $\delta>0$ such that the closure of $V_{3 \delta}$ in $D$ is compact. The proof will be complete once we show that if $V_{\delta} \cap \varphi^{n}\left(V_{\delta}\right) \neq \emptyset$ for some $n \in \mathbb{Z}$, then $\varphi^{n}\left(V_{\delta}\right) \subset V_{3 \delta}$.

Suppose that $\varphi^{n}\left(z_{1}\right)=z_{2}$ for $z_{1}, z_{2} \in V_{\delta}$ and $n \in \mathbb{Z}$. Then, for each $z \in V_{\delta}$,

$$
\begin{aligned}
\left\|\left(\overline{h\left(\varphi^{n}(z)\right)}\right)^{-1} \chi_{\varphi^{n}(z)}-(\overline{h(w)})^{-1} \chi_{w}\right\| \leq & \left\|U_{\varphi^{n}}\left[(\overline{h(z)})^{-1} \chi_{z}-\left(\overline{h\left(z_{1}\right)}\right)^{-1} \chi_{z_{1}}\right]\right\| \\
& +\left\|\left(\overline{h\left(z_{2}\right)}\right)^{-1} \chi_{z_{2}}-(\overline{h(w)})^{-1} \chi_{w}\right\|<3 \delta .
\end{aligned}
$$

Thus $\varphi^{n}\left(V_{\delta}\right) \subset W_{3 \delta}$. Since $\varphi^{n}\left(V_{\delta}\right)$ is connected and intersects $V_{\delta} \subset V_{3 \delta}$, it follows that $\varphi^{n}\left(V_{\delta}\right) \subset V_{3 \delta}$.

The proof is complete.

We shall say that a domain $\Omega$ in $\mathbb{C}^{N}$ has property $\mathscr{B}$ if, for each $w \in \Omega, \chi_{w} \neq 0$ (or, equivalently, $K_{\Omega}(w, w)>0$ ) and the Bergman metric tensor

$$
g_{\Omega}(w)=\sum_{i, j=1}^{N} \frac{\partial^{2}}{\partial z_{i} \partial \bar{z}_{j}} \log K_{\Omega}(w, w) \mathrm{d} z_{i} \mathrm{~d} \bar{z}_{j}
$$

is positive-definite. As is known (cf. [8, p. 296], every domain in $\mathbb{C}^{N}$ which is biholomorphically equivalent to a bounded domain has property $\mathscr{B}$.

Theorem 3.3. Suppose that $D$ has property $\mathscr{B}$. Then $(D, \varphi)$ has property (A).

Proof. Let $d_{D}$ be the geodesic distance relative to $g_{D}$. Let $w$ be a point in $D$. For each $\delta>0$, let $B_{D}(w, \delta)$ denote the open ball relative to $d_{D}$ centered at $w$ with radius $\delta$. It is an elementary result from differential geometry that there is $\delta>0$ such that the closure of $B_{D}(w, 3 \delta)$ in $D$ is compact. To end the proof, it suffices to show that if

for some $n \in \mathbb{Z}$, then

$$
\varphi^{n}\left(B_{D}(w, \delta)\right) \cap B_{D}(w, \delta) \neq \emptyset
$$

$$
\varphi^{n}\left(B_{D}(w, \delta)\right) \subset B_{D}(w, 3 \delta) .
$$

Suppose that $\varphi^{n}\left(z_{1}\right)=z_{2}$ for $z_{1}, z_{2} \in B_{D}(w, \delta)$ and $n \in \mathbb{Z}$. As is well known (cf. $\left[8\right.$, p. 299], $\varphi$ is an isometry with respect to $d_{D}$. Therefore, for each $z \in B_{D}(w, \delta)$,

$$
\begin{aligned}
d_{D}\left(\varphi^{n}(z), w\right) & \leq d_{D}\left(\varphi^{n}(z), \varphi^{n}\left(z_{1}\right)\right)+d_{D}\left(z_{2}, w\right) \\
& =d_{D}\left(z, z_{1}\right)+d_{D}\left(z_{2}, w\right)<3 \delta
\end{aligned}
$$

which yields the desired conclusion.

After this paper had been completed, the author proved that the properties $\mathscr{A}$ and $\mathscr{B}$ are equivalent [3]. Accordingly, Theorem 3.2 is subsumed in Theorem 3.3. Also Theorem 3.1 is a consequence of Theorem 3.3, for if $D$ is a domain in $\mathbb{C}$ such that $L^{2} H(D) \neq\{0\}$, then $D$ has property $\mathscr{B}$ (cf. [3, Thm. 2]). Alternatively, Theorem 3.1 can be deduced from Theorem 3.2 by applying the result of [2]. 


\section{Pure point spectrum (continued)}

Let $\mathscr{H}(D)$ be the space of all holomorphic mappings of $D$ into itself equipped with the compact open topology. With composition as semigroup operation, $\mathscr{H}(D)$ is a topological semigroup. The identity mapping is the identity of $\mathscr{H}(D)$.

Theorem 4.1. Suppose that $(D, \varphi)$ has property (A) and that $U_{\varphi}$ has pure point spectrum. Then the closure of $\left\{\varphi^{k}: k \in \mathbb{Z}\right\}$ in $\mathscr{H}(D)$ is a compact topological group.

Proof. We first show that if the closure $G$ of $\left\{\varphi^{k}: k \in \mathbb{Z}\right\}$ in $\mathscr{H}(D)$ is compact, then it is a topological group. It is clear that $G$ is a topological semigroup. Let $\psi$ be an element of $G$. Since the topology of $\mathscr{H}(D)$ is metrisable, there exists a sequence $\left(k_{n}\right)_{n \in \mathbb{N}}$ in $\mathbb{Z}$ such that $\psi=\lim _{n \rightarrow \infty} \varphi^{k_{n}}$. By the compactness of $G$, there is a subsequence $\left(k_{n_{m}}\right)_{m \in \mathbb{N}}$ of $\left(k_{n}\right)_{n \in \mathbb{N}}$ such that the sequence $\left(\varphi^{-k_{n_{m}}}\right)_{m \in \mathbb{N}}$ converges to an element of $G$. Of course, $\lim _{m \in \infty} \varphi^{-k_{n_{m}}}=\psi^{-1}$. Thus $G$ is a group. Since $G$ is a compact group which is a topological semigroup, it follows from a theorem of Ellis [5] that $G$ is a topological group.

Suppose now that $G$ is not compact. Then, by Montel's theorem, there exists $z \in D$ such that for each open neighbourhood $\Omega$ of $z$, the closure of $\bigcup_{k \in \mathbb{Z}} \varphi^{k}(\Omega)$ in $D$ is not compact. Let $U$ and $V$ be relatively compact neighbourhoods of $z$ in $D$ such that if $U \cap \varphi^{n}(U) \neq \emptyset$ for some $n \in \mathbb{Z}$, then $\varphi^{n}(U) \subset V$. As easy exhaustion argument shows the existence of a maximal subset $\mathscr{M}$ of $\mathbb{Z}$ such that $\varphi^{m}(U) \cap \varphi^{n}(U)=\emptyset$ for distinct $m$ and $n$ in $\mathscr{M}$. We claim that $\mathscr{M}$ is infinite.

In fact, given $k \in \mathbb{Z}$, choose $m \in \mathscr{M}$ so that $\varphi^{m}(U) \cap \varphi^{k}(U) \neq \emptyset$. Then $U \cap$ $\varphi^{k-m}(U) \neq \emptyset$ whence $\varphi^{k-m}(U) \subset V$ and further $\varphi^{k}(U) \subset \varphi^{m}(V)$. Consequently,

$$
\bigcup_{k \in \mathbb{Z}} \varphi^{k}(U) \subset \bigcup_{m \in \mathscr{M}} \varphi^{m}(V)
$$

and as the closure of $\bigcup_{k \in \mathbb{Z}} \varphi^{k}(U)$ in $D$ is not compact, $\mathscr{M}$ must be infinite, as claimed.

Applying now an argument from Example 1.5, we see that $\varphi_{*}$ is not finite. Hence, in view of Theorem 1.3, the spectrum of $U_{\varphi}$ cannot be pure point. This contradiction completes the proof.

As a corollary to Theorem 3.1, 3.2 and 4.1, we obtain

Theorem 4.2. Let $D$ be either a domain in $\mathbb{C}$ or a domain in $\mathbb{C}^{N}(N \geq 2)$ having property $\mathscr{A}$. Then the closure of $\left\{\varphi^{k}: k \in \mathbb{Z}\right\}$ in $\mathscr{H}(D)$ is a compact topological group.

Theorem 4.2 generalizes some results of $[\mathbf{1 1}, \mathbf{1 2}]$. The proofs to the theorems of which Theorem 4.2 is a consequence rely on a modification of an argument due to T. Mazur [12]. Mzaur's original argument used differential geometry and involved an assumption on $D$ stronger than property $\mathscr{B}$, namely property $\mathscr{C}$ which will be introduced below.

Theorem 4.3. Suppose that $D$ has property $\mathscr{A}$. If the orbit of some point in $D$ has compact closure in $D$, then the orbits of all points in $D$ have compact closure in $D$.

The above theorem fails if all the assumptions about $D$ and $\varphi$ are dropped. This is shown by the following

Example 4.4. Let $D=\mathbb{C}^{2}$ and let $\varphi$ be defined as

$$
\varphi\left(z_{1}, z_{2}\right)=\left(z_{1}, z_{1}+z_{2}\right) \quad\left(z_{1}, z_{2} \in \mathbb{C}\right) .
$$

Then all elements of $\{0\} \times \mathbb{C}$ are fixed points for $\varphi$, and for each $w \in \mathbb{C} \backslash(\{0\} \times \mathbb{C})$, the closure $\mathscr{O}(w)$ is not compact. 


\section{Absolutely continuous spectrum}

We shall say that a point $w$ in $D$ is wandering if there exists an open neighbourhood $U$ of $w$ such that $U \cap \varphi^{n}(U)=\emptyset$ for each $n \in \mathbb{N}$. Of course, we may equally well assume in this definition that $U \cap \varphi^{n}(U)=\emptyset$ holds for all $n \in \mathbb{Z} \backslash\{0\}$.

Suppose that $w \in D$ is not wandering, and let $U$ be an open neighbourhood of $w$. Then, $U \cap \varphi^{n}(U) \neq \emptyset$ for infinitely many $n \in \mathbb{N}$. In fact, if there is $n_{0} \in \mathbb{N}$ such that $U \cap \varphi^{n}(U) \neq \emptyset$ for $n \geq n_{0}$, then $w \neq \varphi^{n}(w)$ for $n \geq n_{0}$ and hence for all $n \in \mathbb{N}$. Now, if $V$ is an open neighbourhood of $w$ contained in $U$ such that $V \cap \varphi^{n}(V)=\emptyset$ for $1 \leq n \leq n_{0}$, then, clearly, $V \cap \varphi^{n}(V)=\emptyset$ for all $n \in \mathbb{N}$, a contradiction.

Let

$$
H_{c}=H_{a c} \oplus H_{s c}
$$

be the orthogonal decomposition of $H_{c}$ in which $H_{a c}$ consists of those $f \in H_{c}$ for which the measure $\pi_{f, f}$ is absolutely continuous with respect to the Lebesgue measure in $\mathbb{T}$, and $H_{s c}$ consists of those $f \in H_{c}$ for which the measure $\pi_{f, f}$ is singular with respect to the Lebesgue measure in $\mathbb{T}$. As is known, this decomposition reduces the projection-valued measure $P$.

Theorem 5.1. Suppose that a point $w$ in $D$ is wandering. Then $\chi_{w} \in H_{a c}$.

Proof. Let $U$ be an open neighbourhood of $w$ such that $U \cap \varphi^{n}(U)=\emptyset$ holds for all $n \in \mathbb{Z} \backslash\{0\}$. Then, clearly, $\varphi^{m}(U) \cap \varphi^{n}(U)=\emptyset$ for distinct $m$ and $n$ in $\mathbb{Z}$.

By (1.10) and Levi's monotone convergence theorem,

$$
\int_{U} \sum_{k \in Z}\left|\left(U_{\varphi^{k}} \chi_{w}, \chi_{z}\right)\right|^{2} \mathrm{~d} \lambda(z)=\sum_{k \in Z} \int_{\varphi^{k}(U)}\left|\chi_{w}(z)\right|^{2} \mathrm{~d} \lambda(z) \leq\left\|\chi_{w}\right\|^{2} .
$$

This jointly with (1.2) and Plancherel's theorem shows that for $\lambda$-almost all $z$ in $U$, the measure $\pi_{\chi_{w}, \chi_{z}}$ is absolutely continuous with respect to the Lebesgue measure in $\mathbb{T}$. Since the mapping $D \ni z \mapsto \chi_{z} \in L^{2} H(D)$ is continuous, it follows that for all $z$ in $U$, the measure $\pi_{\chi_{w}, \chi_{z}}$ is absolutely continuous with respect to the Lebesgue measure in $\mathbb{T}$. In particular, this is the case of $\pi_{\chi_{w}, \chi_{w}}$.

The proof is complete.

Theorem 5.2. If there is a wandering point in $D$, then $H_{a c} \neq\{0\}$.

Proof. The set $W$ of wandering points in $D$ is clearly open. If $W \neq \emptyset$, then, in view of (1.6), there exists $w \in W$ such that $\chi_{w} \neq 0$. Now the theorem follows upon applying Theorem 5.1.

Theorem 5.3. Suppose that $(D, \varphi)$ has property (A) and $H_{a c} \neq\{0\}$. Then every point in $D$ is wandering.

Proof. Suppose that $f \in H_{a c} \backslash\{0\}$. Let $w$ be a point in $D$. Let $U$ and $V$ be open neighbourhoods of $w$ with compact closure in $D$ such that if $U \cap \varphi^{n}(U) \neq \emptyset$ for some $n \in \mathbb{Z}$, then $\varphi^{n}(U) \subset V$. Given $z \in V$, let $\chi_{z}=g_{z}^{\prime}+g_{z}^{\prime \prime}$ be the decomposition of $\chi_{z}$ with respect to $H_{p p} \oplus H_{s c}$ and $H_{a c}$. Clearly, $\pi_{f, \chi_{z}}=\pi_{f, g_{z}^{\prime \prime}}$. Hence, in view of (1.3), the measure $\pi_{f, \chi_{z}}$ is absolutely continuous with respect to the Lebesgue measure in $\mathbb{T}$. By (1.2) and the Riemann-Lebesgue lemma,

$$
\lim _{k \rightarrow \pm \infty}\left(U_{\varphi^{k}} f, \chi_{z}\right)=0 .
$$

In view of (1.8), the restriction to $V$ of the functions $z \mapsto\left(U_{\varphi^{k}} f, \chi_{z}\right)(k \in \mathbb{Z})$ are uniformly bounded. Applying Lebesgue's dominated convergence theorem and taking into account (1.10) and (5.1), we get

$$
\lim _{k \rightarrow \pm \infty} \int_{\varphi^{k}(V)}|f|^{2} \mathrm{~d} \lambda=\lim _{k \rightarrow \pm \infty} \int_{V}\left|\left(U_{\varphi^{k}} f, \chi_{z}\right)\right|^{2} \mathrm{~d} \lambda .
$$


Suppose that $w$ is not wandering. Then there exists a sequence $\left(k_{n}\right)_{n \in \mathbb{N}}$ of positive integers such that $\lim _{n \rightarrow \infty} k_{n}=\infty$ and $U \cap \varphi^{k_{n}}(U) \neq \emptyset$ for each $n \in \mathbb{N}$. It is clear that given $n \in \mathbb{N}$, we have $\varphi^{k_{n}}(U) \subset V$. Hence $U \subset \varphi^{-k_{n}}(V)$ and further

$$
\int_{U}|f|^{2} \mathrm{~d} \lambda \leq \int_{\varphi^{-k_{n}(V)}}|f|^{2} \mathrm{~d} \lambda .
$$

The last inequality together with (5.1) yields

$$
\int_{U}|f|^{2} \mathrm{~d} \lambda=0
$$

Since $f \neq 0 \lambda$-a.e. and $\lambda(U)>0$, we get a contradiction.

The proof is complete.

Theorem 5.4. Suppose that $(D, \varphi)$ has property $(\mathrm{A})$ and that $U_{\varphi}$ has a non-zero absolutely continuous component in the spectrum. Then the spectrum of $U_{\varphi}$ is purely absolutely continuous.

Proof. Suppose that $H_{a c} \neq\{0\}$. Then, by Theorem 5.3, every point in $D$ is wandering, and, by Theorem 5.1, $\chi_{w} \in H_{a c}$ for each $w \in D$. In view of (1.7), the Hahn-Banach theorem, and the Riesz theorem, the linear span of $\left\{\chi_{w}: w \in D\right\}$ is dense in $L^{2} H(D)$. Therefore $H_{a c}$ coincides with $L^{2} H(D)$.

The proof is complete.

As a consequence of Theorems 5.2, 5.3, and 5.4, we obtain

Theorem 5.5. Suppose that $(D, \varphi)$ has property $(\mathrm{A})$. If some point in $D$ is wandering, then all points in $D$ are so.

Notice that the above theorem fails if all the assumptions about $D$ and $\varphi$ are dropped. In fact, in $D$ and $\varphi$ are as in Example 4.4, then the set of wandering points in $D$ coincides with $\mathbb{C}^{2} \backslash(\{0\} \times \mathbb{C})$.

We shall say that a domain $\Omega$ has property $\mathscr{C}$ if it has property $\mathscr{B}$ and is complete with respect to the geodesic distance $d_{\Omega}$. As is known (cf. $[\mathbf{9}]$ ), every domain in $\mathbb{C}^{N}$ which is biholomorphically equivalent to a bounded analytic polyhedron has property $\mathscr{C}$.

Theorem 5.6. Suppose that $D$ has property $\mathscr{C}$. Then the spectrum of $U_{\varphi}$ is either pure point or purely absolutely continuous.

Proof. Let $\mathfrak{H}(D)$ be the group of all holomorphic automorphisms of $D$, the group operation being composition of mappings. Since $D$ has property $\mathscr{C}$ and in particular has property $\mathscr{B}$, it follows that under the compact open topology, $\mathfrak{H}(D)$ is a Lie group (cf. $[\mathbf{8}, 300]$ ). Let $G$ be the closure of $\left\{\varphi^{n}: n \in \mathbb{Z}\right\}$ in $\mathfrak{H}(D)$. Clearly, $G$ is a locally compact monothetic group. Therefore, either $G$ is compact or $G=\left\{\varphi^{n}: n \in\right.$ $\mathbb{Z}\}$ and $Z \ni \varphi \mapsto \varphi^{n} \in G$ is topological isomorphism of $\mathbb{Z}$ and $G$ [15, p. 39]. In the first case, by virtue of Theorem $2.1, U_{\varphi}$ has pure point spectrum. In the other case, it turns out that $U_{\varphi}$ has purely absolutely continuous.

Indeed, suppose that $\left\{\varphi^{n}: n \in \mathbb{Z}\right\}$ is topologically isomorphic to $\mathbb{Z}$. If the spectrum of $U_{\varphi}$ is not purely absolutely continuous, then, by Theorems 5.2 and 5.4 , no point in $D$ is wandering. Fix $w \in D$. Since $D$ is complete with respect to $d_{D}$, it follows from the Hopf-Rinov (cf. [8, p. 56]) theorem that for each $\delta>0$, the closure of $B_{D}(w, \delta)$ in $D$ is compact. Choose any $\delta_{0}>0$. Since $w$ is not wandering, there is an unbounded sequence $\left(k_{n}\right)_{n \in \mathbb{N}}$ of positive numbers such that

$$
B_{D}\left(w, \delta_{0}\right) \cap \varphi^{k_{n}}\left(B_{D}\left(w, \delta_{0}\right)\right) \neq \emptyset
$$

for each $n \in \mathbb{N}$. Consequently, for each $\delta \geq \delta_{0}$ and each $n \in \mathbb{N}$,

$$
B_{D}(w, \delta) \cap \varphi^{k_{n}}\left(B_{D}(w, \delta)\right) \neq \emptyset
$$


whence, as in the proof of Theorem 3.3,

$$
\varphi^{k_{n}}\left(B_{D}(w, \delta)\right) \subset B_{D}(w, 3 \delta) .
$$

By Montel's theorem and the fact that each compact subset of $D$ is contained in some $B_{D}(w, \delta)\left(\delta \geq \delta_{0}\right)$, we conclude that the closure of $\left\{\varphi^{k_{n}}: n \in \mathbb{N}\right\}$ in $\mathfrak{H}(D)$ is compact. Since $\left\{\varphi^{n}: n \in \mathbb{Z}\right\}$ is topologically isomorphic to $\mathbb{Z}$, this cannot be the case unless $\left(k_{n}\right)_{n \in \mathbb{N}}$ is bounded. This contradiction completes the proof.

We conclude with a simple application of the theorem established. Let $D$ and $\varphi$ be as in Example 1.5. Then, clearly, $D$ is biholomorphically equivalent to a polydisc which, being a bounded analytic polyhedron, has property $\mathscr{C}$. Since $U_{\varphi}$ has purely continuous spectrum, it follows from Theorem 5.5 that the spectrum of $U_{\varphi}$ is in fact purely absolutely continuous.

\section{Acknowledgement}

The author would like to thank Professor T. Mazur for a stimulating discussion during the IX Conference on Analytic Functions, 1-8 June 1986, Lublin, Poland. The present note was written while the author was staying at McMaster University, Hamilton, Ontario, Canada. The author would like to thank Professor H. Heinig for making the stay possible and for warm hospitality.

\section{References}

[1] S. Bergman, The Kernel Function and Conformal Mapping, in Math. Surveys 5 (Amer. Math. Soc, Providence, R. I., second, revised ed., 1970).

[2] W. Chojnacki, 'On some functionals on Bergman spaces', Bull. Polish Acad. Sci. Math. 37 (1989), 351-353.

[3] W. Chojnacki, 'On the Bergman metric tensor', Bull. Polish Acad. Sci. Math. 39 (1991), 147-150.

[4] Dang-Ngoc-Nghiem, 'On the classification of dynamical systems', Ann. Inst. H. Poincaré Sect. B (N.S.) 9 (1973), 397-425.

[5] R. Ellis, 'Locally compact transformation groups', Duke Math. J. 24 (1957), 119-126.

[6] O. Forster, Lectures on Riemann Surfaces (Springer-Verlag, New York, 1981).

[7] W. H. Gottschalk and G. A. Hedlund, Topological dynamics, in Colloquium Publications 36 (Amer. Math. Soc., Providence, R. I., 1955).

[8] S. Helgason, Differential Geometry and Symmetric Spaces (Academic Press, New York, 1962).

[9] S. Kobayashi, 'Geometry of bounded domains', Trans. Amer. Math. Soc. 92 (1959), 267-290.

[10] K. Maurin, Methods of Hilbert Spaces (Polish Scientific Publishers, Warszawa, 1972).

[11] T. Mazur, 'Spectral properties of automorphisms of the unit dics', Demonstratio Math. 17 (1984), 1069-1072.

[12] T. Mazur, 'Canonical isometry on weighted Bergman spaces', Pacific J. Math. 136 (1989), 303-310.

[13] T. Mazur and M. Skwarczyński, 'Spectral properties of holomorphic automorphism with fixed point', Glasgow Math. J. 28 (1986), 25-30.

[14] W. Parry, Topics in Ergodic Theory (Cambridge University Press, Cambridge, 1981).

[15] W. Rudin, Fourier Analysis on Groups (Interscience, New York, 1962).

[16] J. J. O. O. Wiegerinck, 'Domains with finite dimensional Bergman spaces', Math. Z. 187 (1984), 559-562.

[17] A. Zygmund, Trigonometric Series, I (Cambridge University Press, 1959). 
INSTYTUT MATEMATYKI

UNIWERSYTET WARSZAWSKI

Pąac Kultury i Nauki, IX P.

00-901 WarszaWa, Poland 